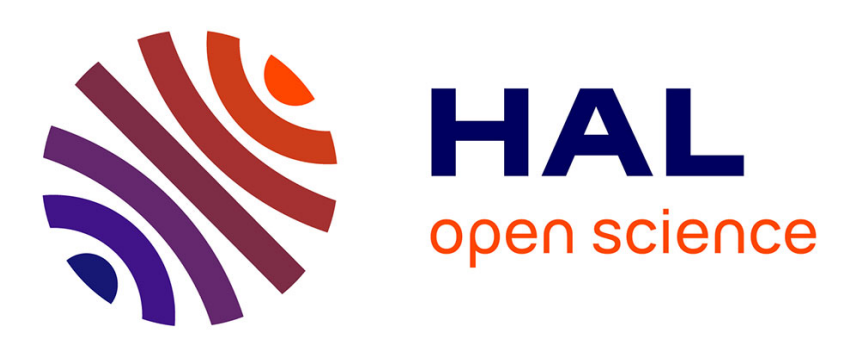

\title{
Schwarz method justification of a coupling between finite elements and integral representation for Maxwell exterior problem
}

\author{
Eric Darrigrand, Nabil Gmati, Rania Rais
}

\section{- To cite this version:}

Eric Darrigrand, Nabil Gmati, Rania Rais. Schwarz method justification of a coupling between finite elements and integral representation for Maxwell exterior problem. Comptes Rendus. Mathématique, 2014, 352 (4), pp.311-315. 10.1016/j.crma.2014.02.006 . hal-00951206

\author{
HAL Id: hal-00951206 \\ https://hal.science/hal-00951206
}

Submitted on 24 Feb 2014

HAL is a multi-disciplinary open access archive for the deposit and dissemination of scientific research documents, whether they are published or not. The documents may come from teaching and research institutions in France or abroad, or from public or private research centers.
L'archive ouverte pluridisciplinaire HAL, est destinée au dépôt et à la diffusion de documents scientifiques de niveau recherche, publiés ou non, émanant des établissements d'enseignement et de recherche français ou étrangers, des laboratoires publics ou privés. 


\title{
Schwarz method justification of a coupling between finite elements and integral representation for Maxwell exterior problem
}

\author{
Eric Darrigrand ${ }^{1}$, Nabil Gmati ${ }^{2}$, Rania Rais ${ }^{1,2}$ \\ ${ }^{1}$ Université de Rennes 1, IRMAR, Campus de Beaulieu, 35042 Rennes, France \\ ${ }^{2}$ Université de Tunis El Manar, Ecole Nationale d'Ingénieurs de Tunis, LAMSIN, B.P. 37, 1002 Le Belvédère, Tunisia
}

Notice: this is the author's version of a work that was accepted for publication in Comptes rendus - Mathématique. Changes resulting from the publishing process, such as peer review, editing, corrections, structural formatting, and other quality control mechanisms may not be reflected in this document. Changes may have been made to this work since it was submitted for publication. A definitive version was subsequently published in C. R. Acad. Sci. Paris, Ser. I (2014), http://dx.doi.org/10.1016/j.crma.2014.02.006

\begin{abstract}
We are interested in the resolution of an exterior Maxwell problem in 3D using a coupling between finite elements and integral representation. This strategy is interpreted as a Schwarz method which suggests a preconditioner for Krylov solvers. Numerical results confirm the relevance of the resolution scheme.

Résumé

Justification par la méthode de Schwarz du couplage entre éléments finis et représentation intégrale pour le problème de Maxwell en domaine extérieur. Nous nous intéressons à la résolution numérique du problème de Maxwell extérieur en $3 D$ par la méthode de couplage entre éléments finis et représentations intégrales. Nous limitons notre étude au cas d'un obstacle de type conducteur parfait. Cette stratégie est interprétée comme une méthode de Schwarz qui suggère un préconditionneur pour les solveurs de Krylov. Par suite, nous menons une étude numérique pour valider la pertinence d'un tel choix.
\end{abstract}

\section{Version française abrégée :}

Nous nous intéressons au problème de diffraction d'ondes électromagnétiques par un conducteur parfait en régime harmonique. Pour résoudre ce problème extérieur, nous considérons la méthode de couplage entre éléments finis et représentations intégrales (problème (2), connue sous la dénomination CEFRI, cf. [3]). Dans ce papier, nous analysons plus spécifiquement une stratégie de résolution itérative du couplage CEFRI inspirée de [4]. Nous montrons dans un premier temps l'analogie entre la méthode CEFRI et la méthode de Schwarz avec recouvrement total pour la résolution du problème de Maxwell en domaine

Email addresses: eric.darrigrand-lacarrieu@univ-rennes1.fr (Eric Darrigrand ${ }^{1}$ ), nabil.gmati@enit.rnu.tn (Nabil Gmati ${ }^{2}$ ), rania.rais@lamsin.rnu.tn (Rania Rais ${ }^{1,2}$ ). 
non borné (problèmes (5) et (6)). Cette réinterprétation de la méthode CEFRI a été initialement présentée dans [1]. Dans le cas d'une géométrie sphérique, elle nous permet d'établir le taux de convergence de la méthode itérative inhérente au problème (2) : la méthode de Schwarz avec recouvrement total converge si le bord $\Gamma$ de l'obstacle est assez éloigné de la frontière artificielle $\Sigma$. Ce résultat est confirmé par des tests numériques réalisés avec des épaisseurs différentes entre $\Gamma$ et $\Sigma$ (voir Fig. 2). Cette relecture de la méthode CEFRI offre également une technique de préconditionnement.

Pour des raisons pratiques de mise en ouvre numérique, la prise en compte de la condition essentielle imposée sur le bord $\Gamma$, se fait par une stratégie de pénalisation qui consiste à remplacer la condition au bord $E \times n_{\gamma}=0$ du problème (2) par $\varepsilon_{p}\left(n_{\gamma} \times \operatorname{curl} E\right)+E \times n_{\gamma}=0$ où $\varepsilon_{p}>0$ est choisi petit. Après discrétisation, cette méthode nous amène à résoudre un système linéaire impliquant une matrice éléments finis classique $A$ ainsi qu'un bloc plein $C$ dû à la représentation intégrale. La matrice du système linéaire à résoudre $A+C$ est mal conditionnée. En appliquant le préconditionneur suggéré par la méthode de Schwarz sur l'équation induite de la discrétisation du problème de diffraction (3), le système devient alors

$$
\left(I+A^{-1} C\right) E=A^{-1} F,
$$

$F$ étant le second membre du système initial.

Un travail numérique de développement de nouveaux outils intégraux dans la librairie éléments finis MÉLINA++ ([5]) a permis la validation de la méthode CEFRI et d'une stratégie itérative de type Krylov. Plusieurs cas tests montrent la convergence éléments finis ainsi que la convergence de la résolution itérative : La figure 3 (gauche) montre l'erreur relative pondérée par la matrice du système linéaire en fonction de la finesse du maillage pour différentes valeurs du paramètre de pénalisation à un nombre d'onde fixé; La figure 3 (droite) montre la convergence du solveur itératif pour différents nombres d'onde.

\section{Introduction}

We are interested in the resolution of the 3D exterior time-harmonic Maxwell equations. To this aim, we focus on a combination of finite elements and integral representation ([3]). This strategy leads to an equivalent problem on a reduced bounded domain delimited by the surface of the scatterer and an artificial boundary with exact artificial boundary condition. No a priori condition is required on the distance between the scatterer and the artificial boundary but a difficult issue consists in the elaboration of a resolution strategy. A relevant idea was suggested in [4]. We propose the interpretation of this idea as an application of the Schwarz method, following the work done in [1] for Helmholtz equation. Hence, the theory on the Schwarz method justifies the use of Krylov solvers and the choice of a preconditioner.

In the next section, we derive the formulation of the system to be solved. Section 2 is devoted to the Schwarz interpretation of the resolution strategy suggested in [4]. In Section 3, the speed of convergence is estimated in the case of a spherical scatterer. Finally, some numerical results illustrate the theoretical properties.

\section{Scattering by a perfect conductor}

Let us consider $\Omega_{i}$ a bounded scatterer in $\mathbb{R}^{3}$ with a regular boundary $\Gamma$ and $\Omega_{e}$ its unbounded complementary. We are concerned with the scattering of a time-harmonic electromagnetic wave by the perfect conductor $\Omega_{i}$. Our purpose is to determine the total field $E=E^{s}+E^{i n c}$ where $E^{i n c}$ is the incident wave and $E^{s}$ is the scattered field, solution to the regularized Maxwell equations with essential boundary 
condition on $\Gamma$ and radiation condition at infinity. Considering an integral representation on an artificial

$\Omega_{e}$

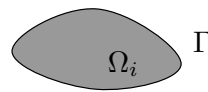

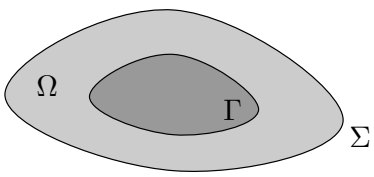

Figure 1. Left: exterior unbounded domain $\Omega_{e}$, right: bounded domain $\Omega$.

boundary $\Sigma$ (see Fig. 1), the exterior problem reduces to a problem on a bounded domain $\Omega$ delimited by $\Gamma$ and $\Sigma$ (see [3]): Find $E$ such that

$$
\left\{\begin{array}{l}
\operatorname{curl} \operatorname{curl} E-t^{-1} \nabla(\operatorname{div} E)-k_{s}^{2} E=0 \text { in } \Omega, \\
E \times n_{\gamma}=0, \operatorname{div} E=0 \text { on } \Gamma, \\
T_{\nu_{1}}(E)=T_{\nu_{1}}\left(E^{i n c}-\mathcal{I}_{\Gamma}(E)\right) \text { and } N_{\nu_{2}}(E)=N_{\nu_{2}}\left(E^{i n c}-\mathcal{I}_{\Gamma}(E)\right) \text { on } \Sigma,
\end{array}\right.
$$

where $n_{\gamma}$ is the exterior unit normal of the domain $\Omega_{i}$ on $\Gamma$; the regularization term $t^{-1} \nabla(\operatorname{div} E)$ allows the use of a Galerkin finite-elements method (see [3]) and the regularization parameter $t^{-1}$ depends on the permittivity and the permeability of the air; $k_{s}$ is the wave number; $\nu_{1}$ and $\nu_{2}$ are complex numbers which have a negative imaginary part. The two operators $T_{\nu_{1}}$ and $N_{\nu_{2}}$ are defined by $T_{\nu_{1}} E=$ $\operatorname{curl} E \times n_{\sigma}+\nu_{1} n_{\sigma} \times\left(E \times n_{\sigma}\right)$ and $N_{\nu_{2}} E=\operatorname{div} E+\nu_{2} E \cdot n_{\sigma}$ with $n_{\sigma}$ the exterior unit normal of the domain $\Omega$ on $\Sigma$. The boundary conditions on $\Sigma$ are derived from the integral representations satisfied by the scattered field and identified by the following expression ([3]): for $x \in \Omega_{e}$,

$\mathcal{I}_{\Gamma}(E)(x)=-k_{s}^{2} \int_{\Omega} \mathcal{R} \mathcal{G}_{t}(x,) E+.\int_{\Omega} \operatorname{curl} \mathcal{R} \mathcal{G}_{t}(x,.) \operatorname{curl} E+t^{-1} \int_{\Omega} \operatorname{div} \mathcal{R} \mathcal{G}_{t}(x, .)^{T} \operatorname{div} E-t^{-1} \int_{\Gamma} \operatorname{div} \mathcal{G}_{t}(x, .)^{T}\left(E \cdot n_{\gamma}\right) d \gamma$, where $\mathcal{G}_{t}=G_{k_{s}} I+\frac{1}{k_{s}^{2}} \operatorname{Hess}\left(G_{k_{s}}-G_{k_{p}}\right)$ is the outgoing Green tensor associated with the differential operator curl curl $-t^{-1} \nabla(\operatorname{div})-k_{s}^{2} I$ of the regularized Maxwell equation; $I$ is the identity matrix in $\mathbb{R}^{3}$; Hess stands for Hessian operator; $k_{p}=\sqrt{t} k_{s}$ and $G_{k}$ is the fundamental solution of Helmholtz equation; $\mathcal{R}$ is a linear operator that maps every regular function $\varphi$ defined on $\Gamma$ into a regular function $\mathcal{R} \varphi$ defined on $\Omega$ which satisfies $\mathcal{R} \varphi=\varphi$ on $\Gamma$ and $\mathcal{R} \varphi=0$ on $\Sigma$. The consideration of the Hilbert space

$$
\mathcal{H}_{t}=\left\{E \in H(\operatorname{curl}, \Omega) / \operatorname{div} E \in L^{2}(\Omega), E \times n_{\gamma}=0 \text { on } \Gamma, E \times n_{\sigma} \in L^{2}(\Sigma)^{3}, E \cdot n_{\sigma} \in L^{2}(\Sigma)\right\},
$$

enables one to write a variational formulation of the problem (2): Find $E \in \mathcal{H}_{t}$ such that

$$
\left(\mathcal{A}_{t}+\mathcal{C}_{t}\right) E=F_{t},
$$

where the operators $\mathcal{A}_{t}$ and $\mathcal{C}_{t}: \mathcal{H}_{t} \rightarrow \mathcal{H}_{t}$ are defined as follows

$$
\begin{aligned}
& \left(\mathcal{A}_{t} E, E^{\prime}\right)_{t}=\int_{\Omega}\left(\operatorname{curl} E \cdot \operatorname{curl} E^{\prime}+t^{-1} \operatorname{div} E \operatorname{div} E^{\prime}-k_{s}^{2} E \cdot E^{\prime}\right)+\int_{\Sigma}\left(\nu_{1}\left(n_{\sigma} \wedge E\right) \cdot\left(n_{\sigma} \wedge E^{\prime}\right)+t^{-1} \nu_{2}\left(n_{\sigma} \cdot E\right)\left(n_{\sigma} \cdot E^{\prime}\right)\right) d \sigma, \\
& \left(\mathcal{C}_{t} E, E^{\prime}\right)_{t}=\int_{\Sigma} T_{\nu_{1}}\left(\mathcal{I}_{\Gamma}(E)\right) \cdot E^{\prime} d \sigma+t^{-1} \int_{\Sigma} N_{\nu_{2}}\left(\mathcal{I}_{\Gamma}(E)\right)\left(n_{\sigma} \cdot E^{\prime}\right) d \sigma,
\end{aligned}
$$

and $F_{t}$ is given by $\left(F_{t}, E^{\prime}\right)_{t}=\int_{\Sigma}\left(T_{\nu_{1}}\left(E^{i n c}\right) \cdot E^{\prime}+t^{-1} N_{\nu_{2}}\left(E^{i n c}\right)\left(n_{\sigma} \cdot E^{\prime}\right)\right) d \sigma$, where $(\cdot, \cdot)_{t}$ is the scalar product on $\mathcal{H}_{t}$.

The problem (3) is well posed as explained in [3] and the operator $\mathcal{A}_{t}$ is invertible. 


\section{Schwarz method interpretation}

In order to solve the system (3), Jin and Liu [4] suggested to consider $\mathcal{C}_{t}$ in the right hand side. An application of the fixed point algorithm leads to finding $E_{n+1}$ such that

$$
\left\{\begin{array}{l}
\operatorname{curl} \operatorname{curl} E_{n+1}-t^{-1} \nabla\left(\operatorname{div} E_{n+1}\right)-k_{s}^{2} E_{n+1}=0 \text { in } \Omega, \\
E_{n+1} \times n_{\gamma}=0, \operatorname{div} E_{n+1}=0 \text { on } \Gamma, \\
T_{\nu_{1}}\left(E_{n+1}\right)=T_{\nu_{1}}\left(E^{i n c}-\mathcal{I}_{\Gamma}\left(E_{n}\right)\right) \text { and } N_{\nu_{2}}\left(E_{n+1}\right)=N_{\nu_{2}}\left(E^{i n c}-\mathcal{I}_{\Gamma}\left(E_{n}\right)\right) \text { on } \Sigma .
\end{array}\right.
$$

In this paper, we focus on an original mathematical justification of convergence of the algorithm expressed by Jin and Liu. We interprete the algorithm defined by (4) as a Schwarz method. This interpretation has been initially proposed for the case of Helmholtz equation in [1]. The strategy is designed by the Total Overlapping Schwarz Method. Indeed the overlapping area is the total domain $\Omega$. We hereby extend their work to the case of Maxwell equations: it consists in replacing equivalently the problem (4) by the two following subproblems. The first one is a transmission problem:

$$
\left\{\begin{array}{l}
\operatorname{curl} \operatorname{curl} E_{2 n+1}-t^{-1} \nabla\left(\operatorname{div} E_{2 n+1}\right)-k_{s}^{2} E_{2 n+1}=0 \text { in } \Omega_{i} \cup \Omega_{e}, \\
n_{\gamma} \times\left[E_{2 n+1}\right]=0, n_{\gamma} \times\left[\operatorname{curl} E_{2 n+1}\right]=-n_{\gamma} \times \operatorname{curl} E_{2 n} \text { on } \Gamma, \\
{\left[\operatorname{div} E_{2 n+1}\right]=0, n_{\gamma} \cdot\left[E_{2 n+1}\right]=-n_{\gamma} \cdot E_{2 n} \text { on } \Gamma,} \\
\lim _{\rho \rightarrow \infty} \int_{\|x\|=\rho}\left\|\operatorname{curl} E_{2 n+1}^{s} \times n_{\gamma}-i k_{s} n_{\gamma} \times\left(E_{2 n+1}^{s} \times n_{\gamma}\right)\right\|^{2} d \gamma=0, \\
\lim _{\rho \rightarrow \infty} \int_{\|x\|=\rho}\left|\sqrt{t^{-1}} \operatorname{div} E_{2 n+1}^{s}-i k_{s} E_{2 n+1}^{s} \cdot n_{\gamma}\right|^{2} d \gamma=0 .
\end{array}\right.
$$

The second one consists in finding $E_{2 n+2}$ such that

$$
\left\{\begin{array}{l}
\operatorname{curl} \operatorname{curl} E_{2 n+2}-t^{-1} \nabla\left(\operatorname{div} E_{2 n+2}\right)-k_{s}^{2} E_{2 n+2}=0 \text { in } \Omega, \\
E_{2 n+2} \times n_{\gamma}=0, \operatorname{div} E_{2 n+2}=0 \text { on } \Gamma \\
T_{\nu_{1}}\left(E_{2 n+2}\right)=T_{\nu_{1}}\left(E_{2 n+1}\right) \text { and } N_{\nu_{2}}\left(E_{2 n+2}\right)=N_{\nu_{2}}\left(E_{2 n+1}\right) \text { on } \Sigma .
\end{array}\right.
$$

The solution $E_{2 n+1}$ of (5) has an explicit solution given by an integral representation. By inserting this representation in the second condition of (6) we effectively obtain the solution of (4). At the iteration $n$, the Schwarz algorithm is defined by $\mathcal{A}_{t} E_{n+1}=-\mathcal{C}_{t} E_{n}+F_{t}$. Numerically, we use the scheme suggested by Jin and Liu and do not use the subproblems (5) and (6). The intermediate problems (5) and (6) are used for theoretical justifications. This enables one to derive convergence estimations that cannot be obtained directly from the system (4). In Section 3, we investigate an analytical calculation of the rate of convergence of the Schwarz method in a spherical configuration.

\section{Analytical estimation of the convergence for a spherical scatterer}

In the case where $\Omega_{i}$ is a perfectly conducting ball, we investigate the rate of convergence of the Total Overlapping Schwarz method. Let us consider the scatterer to be a ball of radius $R_{*}$. We suppose that the artificial boundary $\Sigma$ is a sphere concentric to $\Gamma$ with radius $R>R_{*}$. We first introduce some notations: We denote by $j_{l}$ the spherical Bessel function of degree $l$, by $h_{l}$ the spherical Hankel function of the first kind of degree $l$ and $H_{l}(r)=h_{l}(r)+r h_{l}^{\prime}(r), J_{l}(r)=j_{l}(r)+r j_{l}^{\prime}(r)$. We introduce the tangential vector 
spherical harmonics on the unit sphere $S_{1}, U_{l m}=(1 / \sqrt{l(l+1)}) \nabla Y_{l}^{m}$ and $V_{l m}=n_{\gamma} \times U_{l m}$, where $Y_{l}^{m}$, $l>0, m=-l, \ldots, l$ are the orthonormal scalar spherical harmonics (complete basis of $\left.L^{2}\left(S_{1}\right)\right)$. The sets of $U_{l m}$ and $V_{l m}$ form a complete orthonormal basis for $T^{2}\left(S_{1}\right):=\left\{a: S_{1} \rightarrow \mathbb{C}^{3} / a \in\left(L^{2}\left(S_{1}\right)\right)^{3}, a \cdot n_{\gamma}=0\right\}$.

We define the error $\left(w_{n}\right)_{n}$ on the field $E$ at each iteration by:

$$
w_{2 n+1}=E_{2 n+1}-E \text { in } \Omega_{e}, \quad w_{2 n+1}=E_{2 n+1} \text { in } \Omega_{i}, \quad w_{2 n+2}=E_{2 n+2}-E \text { in } \Omega .
$$

For all $n$, we define $\Lambda_{n}=T_{\nu_{1}}\left(w_{2 n}\right)$ and $\delta_{n}=N_{\nu_{2}}\left(w_{2 n}\right)$. In order to prove that the error $\left(w_{n}\right)_{n}$ converges to zero, we first show that $\Lambda_{n+1}=K \Lambda_{n}$ and $\delta_{n+1}=L \delta_{n}$ with $K: T^{2}\left(S_{1}\right) \rightarrow T^{2}\left(S_{1}\right)$ and $L: L^{2}\left(S_{1}\right) \rightarrow$ $L^{2}\left(S_{1}\right)$ two linear maps. $K$ (resp. $L$ ) has a diagonal representation in the basis $\left(U_{l m}, V_{l m}\right)_{l m}$ of $T^{2}\left(S_{1}\right)$ (resp. $Y_{l}^{m}$ of $L^{2}\left(S_{1}\right)$ ). Let us denote by $\tau_{1, l m}, \tau_{2, l m}$ (resp. $\tau_{3, l m}$ ) the eigenvalues of $K$ (resp. $L$ ). These eigenvalues define the rate of convergence of the Total Overlapping Schwarz method. Taking into account the boundary and transmission conditions, we obtain:

$$
\begin{gathered}
\tau_{1, l m}=\left(1-\frac{H_{l}\left(k_{s} R_{*}\right)}{J_{l}\left(k_{s} R_{*}\right)} \frac{J_{l}\left(k_{s} R\right)-i k_{s} R j_{l}\left(k_{s} R\right)}{H_{l}\left(k_{s} R\right)-i k_{s} R h_{l}\left(k_{s} R\right)}\right)^{-1}, \\
\tau_{2, l m}=\left(1-\frac{h_{l}\left(k_{s} R_{*}\right)}{j_{l}\left(k_{s} R_{*}\right)} \frac{J_{l}\left(k_{s} R\right)-i k_{s} R j_{l}\left(k_{s} R\right)}{H_{l}\left(k_{s} R\right)-i k_{s} R h_{l}\left(k_{s} R\right)}\right)^{-1}, \quad \tau_{3, l m}=\left(1-\frac{H_{l}\left(k_{s} R_{*}\right)}{J_{l}\left(k_{s} R_{*}\right)} \frac{j_{l}\left(k_{s} R\right)}{h_{l}\left(k_{s} R\right)}\right)^{-1} .
\end{gathered}
$$

The convergence of the Total Overlapping Schwarz method is ensured if $\left|\tau_{i, l m}\right|<1, \forall i=1, \ldots, 3, \forall l$. The reader can see that these eigenvalues are independent of the parameter $m$. For $R_{*}=1$, the asymptotic behavior of the spherical Bessel functions for large $l$ leads to the asymptotic estimation $\tau_{i, l m} \sim\left(1-R^{2 l}\right)^{-1}$, $i=1, \ldots, 3$. As a consequence, for small values of $R$, there exists a finite number of coefficients $\tau_{i, l m}$ outside of the unit disk, and for sufficiently large values of $R$, all the coefficients $\tau_{i, l m}$ are in the interior to the unit disk. We conclude that the Schwarz method has a linear convergence for $R$ large enough. The numerical tests illustrate this theoretical result. In Fig. 2, we consider $R_{*}=1$ and $R=R_{*}+e$ with different values of $e: \lambda / 100, \lambda / 10$ or $\lambda / 5$. The cases $e=\lambda / 100$ and $e=\lambda / 10$ exhibit some coefficients larger than 1 while the maximum value of $\left|\tau_{2, l m}\right|$ is strictly lower than 1 at the considered wavenumbers for the thickness $e=\lambda / 5$ but the results are strongly dependent on the wavenumber. Similar asymptotic observations can be done on $\left|\tau_{1, l m}\right|$ and $\left|\tau_{3, l m}\right|$. As a consequence, a Krylov method is a relevant alternative to the algorithm defined by (4): due to the properties of Krylov solvers demonstrated in [2], the convergence of a Krylov method is ensured for the resolution of the problem (3) using $\mathcal{A}_{t}$ as a preconditioner.
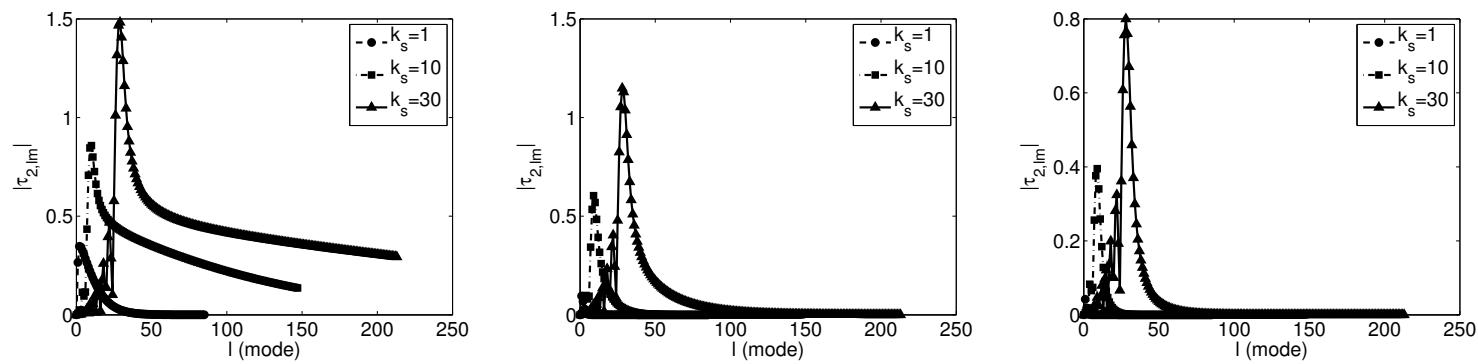

Figure 2. Modulus of $\tau_{2, l m}$ for thickness $e=\lambda / 100$ (left), $\lambda / 10$ (center) and $\lambda / 5$ (right). Cases $k_{s}=1,10$ or 30 .

\section{Preconditioner for a Krylov solver}

The previous work suggests the use of $\mathcal{A}_{t}$ as a preconditioner to solve the problem (3) using a Krylov solver. We hereby consider the resolution of problem (3) using the biconjugate gradient stabilized method. After a finite-elements discretization, the linear system is written under the form $(A+C) E=F$ and the 
preconditioned system becomes $\left(I+A^{-1} C\right) E=A^{-1} F$ where the matrix $C$ involves the integral operators and the matrix $A$ related to the differential operators involves a term resulting from the essential condition considered by a penalization strategy: $\varepsilon_{p}\left(n_{\gamma} \times \operatorname{curl} E\right)+E \times n_{\gamma}=0$ on $\Gamma$, with $\varepsilon_{p}>0$.

The numerical implementation were done using and developing new integrands in the library MÉLINA++ [5]. In this section, we validate the efficiency of the preconditioning strategy by the consideration of an intermediate problem the solution of which is known:

$$
\left\{\begin{array}{l}
\operatorname{curl} \operatorname{curl} E-\nabla(\operatorname{div} E)-k_{s}^{2} E=0 \text { in } \Omega_{e}, \\
E \times n_{\gamma}=\mathcal{G}_{1}^{1} \times n_{\gamma}, \operatorname{div} E=\operatorname{div} \mathcal{G}_{1}^{1} \text { on } \Gamma, \\
\lim _{\rho \rightarrow \infty} \int_{\|x\|=\rho}\left\|\operatorname{curl} E^{s} \times n_{\gamma}-i k_{s} n_{\gamma} \times\left(E^{s} \times n_{\gamma}\right)\right\|^{2} d \gamma=0, \\
\lim _{\rho \rightarrow \infty} \int_{\|x\|=\rho}\left|\operatorname{div} E^{s}-i k_{s} E^{s} \cdot n_{\gamma}\right|^{2} d \gamma=0,
\end{array}\right.
$$

where $\mathcal{G}_{1}^{1}$ is the first vector component of $\mathcal{G}_{1}$. The scatterer is the unit sphere and the artificial boundary $\Sigma$ is the sphere concentric to $\Gamma$ with radius $R=1.5$.
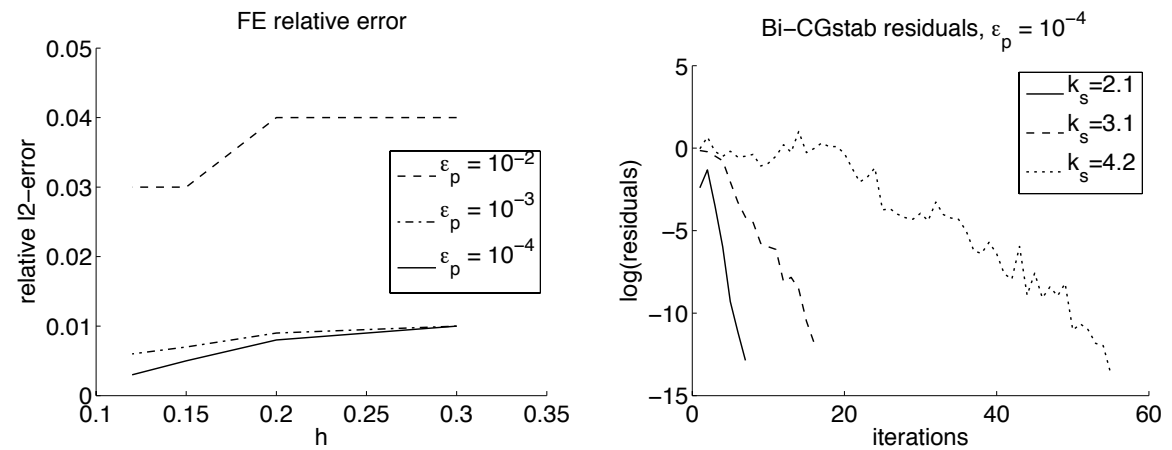

Figure 3. Relative $l^{2}$-error with respect to the discretization (left), behavior of the residuals, case $k_{s} h=2 \pi / 10$ (right)

Fig. 3-left shows the convergence of the relative error with respect to the finite-elements discretization: the relative error is plotted with respect to the average size of the mesh elements, for different values of the penalization parameter $\varepsilon_{p}$, with $k_{s}=3$. Fig. 3-right illustrates a superlinear convergence of the biconjugate gradient stabilized solver applied to the preconditioned system for different values of the wavenumber with $\varepsilon_{p}=10^{-4}$.

\section{References}

[1] F. Ben Belgacem, M. Fournié, N. Gmati, F. Jelassi, "On the Schwarz algorithms for the elliptic exterior boundary value problems", ESAIM: M2AN, vol.39, n. 4, pp. 693-714, 2005.

[2] N. Gmati, B. Philippe, "Comments on the GMRES Convergence for Preconditioned Systems", Large-Scale Scientific Computing, Lecture Notes in Computer Science, vol 4818, pp. 40-51, 2008.

[3] C. Hazard, M. Lenoir, "On the solution of time-harmonic scattering problems for Maxwell's equations", SIAM J. MATH. ANAL., vol. 27, n. 6, pp. 1597-1630, nov. 1996.

[4] J-M. Jin, J. Liu, "A novel hybridization of higher order finite element and boundary integral methods for electromagnetic scattering and radiation Problems", IEEE Transactions on antennas and propagation, vol. 49, n. 12, pp. 1794-1806, dec. 2001.

[5] D. Martin, "http://anum-maths.univ-rennes1.fr/melina/melina++_distrib/", Université Rennes 1, nov. 2013. 